\title{
Microcanonical Transfer Matrix Study of the $Q$-state Potts Model
}

\author{
Richard J. Creswick and Seung-Yeon Kim \\ Department of Physics and Astronomy, University of South Carolina, Columbia, South Carolina 29208, USA
}

\begin{abstract}
The microcanonical transfer matrix is used to study the zeros of the partition function of the $Q$-state Potts model. Results are presented for the Yang-Lee zeros of the 3-state model, the Fisher zeros of the 3-state model in an external field $H_{q}<0$, and the spontaneous magnetization of the 2-state model. In addition, we are able to calculate the ground-state entropy of the 3 -state model and find $s_{0}=0.43153(3)$ in excellent agreement with the exact value, $0.43152 \ldots$
\end{abstract}

\section{Introduction}

The $Q$-state Potts model[1] in two dimensions exhibits a rich variety of critical behavior and is very fertile ground for the analytical and numerical investigation of first- and second-order phase transitions. With the exception of the $Q=2$ Potts (Ising) model in the absence of an external magnetic field [2], exact solutions for arbitrary $Q$ are not known. However, some exact results have been established for the ferromagnetic Potts model [1]. For $Q \leq 4$ there is a second-order phase transition, while for $Q>4$ the transition is first order.

The bond-energy for the $Q$-state Potts model is (in dimensionless units)

$$
E=\sum_{<i, j>}\left[1-\delta\left(\sigma_{i}, \sigma_{j}\right)\right]
$$

where $\langle i, j\rangle$ indicates a sum over nearest-neighbor pairs, $\sigma_{i}=0, \ldots, Q-1$, and $E$ is a positive integer $0 \leq$ $E \leq N_{b}$, where $N_{b}$ is the number of bonds on the lattice. In addition to the energy there are $Q$ order parameters

$$
M_{q}=\sum_{k} \delta\left(\sigma_{k}, q\right),
$$

where $q$ is a fixed integer between 0 and $Q-1$. Note that $0 \leq M_{q} \leq N_{s}$ is also an integer and $N_{s}$ is the number of sites on the lattice.

If we denote the density of states with energy $E$ by $\Omega(E)$, then the partition function for the Potts model is

$$
Z(y)=\sum_{E} \Omega_{Q}(E) y^{E},
$$

where $y=e^{-\beta}$. From (3) it is clear that $Z(y)$ is simply a polynomial in $y$. If we wish to study the partition function in an external field which couples to the order paramter, (2), then one needs to enumerate the states with fixed energy $E$ and fixed order parameter $M, \Omega(M, E)$. The partition function in a magnetic field $H_{q}$ is again a polynomial given by

$$
Z(x, y)=\sum_{M=0}^{N_{s}} \sum_{E=0}^{N_{b}} \Omega_{Q}(M, E) x^{M} y^{E}
$$

where $x=e^{\beta H_{q}}$.

\section{Microcanonical transfer matrix}

By the microcanonical transfer matrix $(\mu \mathrm{TM})[3]$ it is possible to obtain exact integer values for $\Omega(E)$ and $\Omega(M, E)$. Here we describe briefly the $\mu \mathrm{TM}$ on an $L \times$ $L$ lattice with cylindrical boundary conditions. First, an array, $\omega$, which is indexed by the energy $E$ and $L$ variables $\sigma_{i}, 0 \leq i \leq L-1$ for the first row of sites is initialized as

$$
\omega\left(E, \sigma_{0}, \sigma_{1}, \ldots, \sigma_{L-1}\right)=\delta\left(E-\sum_{i=0}^{L-1} h\left(\sigma_{i}, \sigma_{i+1}\right)\right),
$$

where $h(q, p)$ is the contribution to the energy from a single bond.

Following the Binder algorithm河, each spin in the row is traced over in turn, introducing a new spin variable from the next row,

$$
\omega\left(E ; \sigma_{0}^{\prime}, \ldots, \sigma_{L-1}\right)=\sum_{\sigma_{0}} \omega\left(E-h\left(\sigma_{0}, \sigma_{0}^{\prime}\right) ; \sigma_{0}, \ldots, \sigma_{L-1}\right) .
$$

This procedure is repeated until all the spins in the first row have been traced over, leaving a new function of the $L$ spins in the second row. The horizontal bonds connecting the spins in the second row are then taken into account by shifting the energy,

$\omega\left(E ; \sigma_{0}^{\prime}, \ldots, \sigma_{L-1}^{\prime}\right)=\omega\left(E-\sum_{i=0}^{L-1} h\left(\sigma_{i}^{\prime}, \sigma_{i+1}^{\prime}\right) ; \sigma_{0}^{\prime}, \ldots, \sigma_{L-1}^{\prime}\right)$.

This procedure is then applied to each row in turn until the final row is reached. The density of states is then given by

$$
\Omega(E)=\sum_{\sigma_{0}^{\prime}} \sum_{\sigma_{1}^{\prime}} \ldots \sum_{\sigma_{L-1}^{\prime}} \omega\left(E ; \sigma_{0}^{\prime}, \sigma_{1}^{\prime}, \ldots, \sigma_{L-1}^{\prime}\right) .
$$

\section{Partition function zeros}

The analytic structure of the partition function is completely determined by its Yang-Lee (YL) zeros in the complex $x$ plane[5] and its Fisher zeros in the complex $y$ 


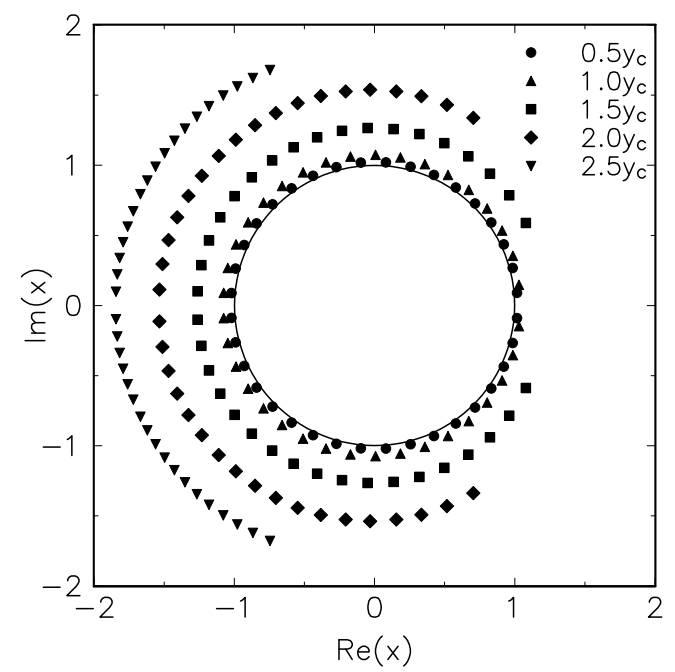

Figure 1: Zeros of a $6 \times 6$ three-state Potts model in the complex $x$ plane for several values of $y$ (cylindrical boundary conditions).

plane 6 . Using the $\mu \mathrm{TM}$ we have studied the YL zeros of the two-dimensional Potts model for $Q=2$, for which the circle theorem of Lee and Yang [5] applies, and, for the first time, for $Q>2[7]$. Figure 1 shows the YL zeros for the three-state Potts model. At $y=0.5 y_{c}$ the zeros are uniformly distributed close to the unit circle. As the temperature is increased the edge singularity moves away from the real axis and the zeros detach from the unit circle. Finally, as $y$ approaches unity, the zeros converge on the point $x=1-Q[7]$. The $Q$-state model in an external field $H_{q}<0$ is in the same symmetry class as the $Q-1$ state model. We have studied the Fisher zeros of the 3-state model as a function of $H_{q} \mathbb{8}$ and determined the critical point and exponents. The critical line is shown in Figure 2.

By introducing the density of zeros $g(\theta, y)$ Lee and Yang[5] showed that the spontaneous magnetization of the Ising model is given by

$$
m_{0}(y)=2 \pi g(0, y) .
$$

Even though they have calculated the density of zeros of the one-dimensional Ising model and confirmed the well-known fact that the model does not exhibit ferromagnetism, the exact form of the density of zeros is not known for $d \geq 2$. We have studied the density of zeros of the two-dimensional Ising model numerically [9], and using (9) we have calculated the spontaneous magnetization (Figure 3 ) which agrees very well with the exact result 2].

\section{Ground-state entropy}

The antiferromagnetic Potts model (APM) is much less well understood than its ferromagnetic counterpart.

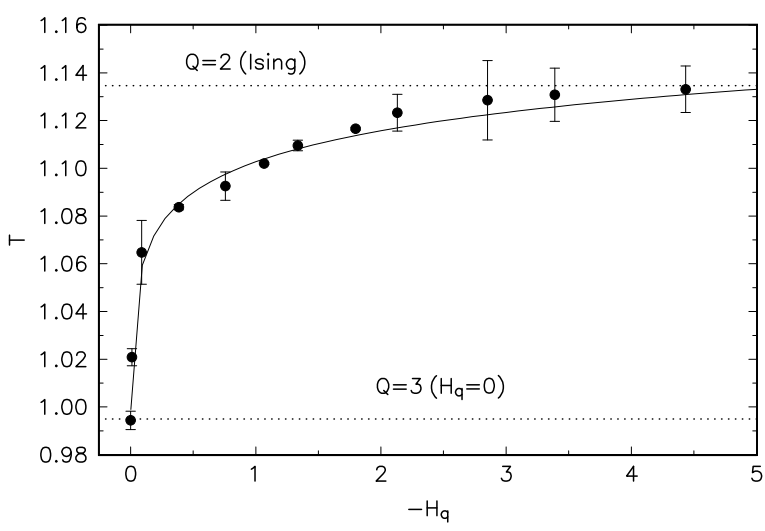

Figure 2: Critical temperatures of the three-state Potts ferromagnet as a function of the magnetic field. The upper dotted line is the Ising transition temperature in the limit $H_{q} \rightarrow-\infty$, while the lower dotted line shows the critical temperature of the three-state Potts model for $H_{q}=0$.

One of the most interesting properties [10] of this model is that for $Q>2$ the ground-state is highly degenerate and the ground-state entropy is nonzero. However, the exact ground-state entropy of the APM is not known except for $Q=3$, where the ground-state entropy per site is 11

$$
s_{0}=\frac{3}{2} \ln \frac{4}{3}=0.43152 \ldots
$$

From the density of states we have obtained the exact ground-state entropy for finite lattices. Table 1 shows the ground-state degeneracy $\Omega_{0}(L)$ of the $L \times$ $L$ three-state APM. The ground-state entropy is then given by

$$
s_{0}(L)=\frac{\ln \Omega_{0}(L)}{N_{s}} .
$$

By using the Bulirsch-Stoer (BST) algorithm[12], we have extrapolated our results for finite lattices to infinite size. The BST estimate for $Q=3$ is $s_{0}=0.43153(3)$, in excellent agreement with (10).

\section{Discussion}

Using the $\mu \mathrm{TM}$, we have calculated the restricted density of states $\Omega(M, E)$ from which the partition function at any temperature and in an arbitrary magnetic field can be evaluated. We have studied the Yang-Lee zeros of the 3-state model and demonstrated that, unlike the Ising model, the locus of zeros is not a circle. Following Lee and Yang we have used the density of zeros to calculate the spontaneous magnetization for the 2 -state model and we are currently extending this work 


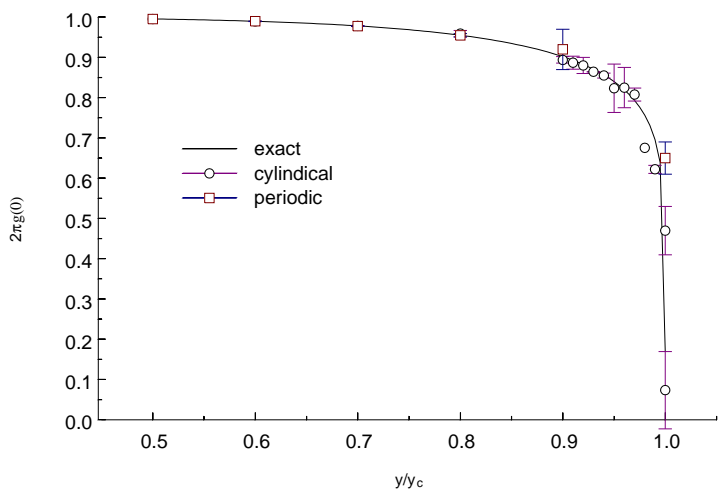

Figure 3: Extrapolated density of zeros as a function of $y$. The solid curve is Yang's exact result for the spontaneous magnetization. Open circles are our results for cylindrical boundary conditions, and open squares are for periodic boundary conditions.

Table 1: The ground-state degeneracy of the three-state APM for even-size lattices (cylindrical boundary conditions).

\begin{tabular}{rr}
\hline$L$ & $\Omega_{0}(L)$ \\
\hline 4 & 4626 \\
6 & 37284186 \\
8 & 9527634436194 \\
10 & 77048019386429374638 \\
12 & 19698820973096872077077373450 \\
14 & 159147870862104841838351532192943853490 \\
\hline
\end{tabular}

to $Q>2$. Since the zeros of the partition function encompass both the ferromagnetic and antiferromagnetic regimes, we are currently investigating the critical behavior of the antiferromagnetic Potts model. Also, the $\mu \mathrm{TM}$ is easily extended to three-dimensional lattices, and we are now extending all of the above calculations to cubic lattices.

Recently it has been suggested [13] that the microcanonical entropy may exhibit weaker finite-size effects than the free energy. The microcanonical ensemble also facilitates the study of phase coexistence and the study of phase transformations in cluster and nuclear physics [13]. The $\mu \mathrm{TM}$ method yields exact values for the microcanonical entropy of finite systems allowing us to study finite size effects in the microcanonical ensemble.

\section{References}

[1] R. B. Potts, Proc. Camb. Phil. Soc. 48 (1952) 106; F. Y. Wu, Rev. Mod. Phys. 54 (1982) 235; R. J. Creswick and S.-Y. Kim, cond-mat/9701018; J. Phys. A 30 (1997) 8785, and references therein.

[2] L. Onsager, Phys. Rev. 65 (1944) 117; C. N. Yang, ibid. 85 (1952) 808.

[3] B. Stošić, S. Milošević, and H. E. Stanley, Phys. Rev. B 41 (1990) 11466; G. Bhanot, M. Creutz, and J. Lacki, Phys. Rev. Lett. 69 (1992) 1841; R. J. Creswick, Phys. Rev. E 52 (1995) 5735.

[4] K. Binder, Physica 62 (1972) 508.

[5] C. N. Yang and T. D. Lee, Phys. Rev. 87 (1952) 404; T. D. Lee and C. N. Yang, ibid. 410.

[6] M. E. Fisher, in: Lectures in Theoretical Physics, vol. 7c, W. E. Brittin, ed. (University of Colorado Press, Boulder, 1965) p. 1.

[7] S.-Y. Kim and R. J. Creswick, Phys. Rev. Lett. 81 (1998) 2000.

[8] S.-Y. Kim and R. J. Creswick, cond-mat/9806294 (Phys. Rev. E, in press).

[9] R. J. Creswick and S.-Y. Kim, Phys. Rev. E 56 (1997) 2418.

[10] R. Shrock and S.-H. Tsai, Phys. Rev. E 55 (1997) 5165; S.-H. Tsai, ibid. 57 (1998) 2686, references therein.

[11] E. Lieb, Phys. Rev. 162 (1967) 162.

[12] R. Bulirsch and J. Stoer, Numer. Math. 6 (1964) 413; M. Henkel and G. Schütz, J. Phys. A 21 (1988) 2617.

[13] A. Hüller, Z. Phys. B 95 (1994) 63; M. Kastner, M. Promberger, and A. Hüller, in: Computer Simulation Studies in Condensed-Matter Physics, vol. 11, D. P. Landau and H.-B. Schüttler, eds. (Springer, Berlin, in press); D. H. E. Gross, Phys. Rep. 279 (1997) 119, and references therein. 